\title{
Morphological transformation of gold nanoparticles on graphene oxide: effects of capping ligands and surface interactions
}

\author{
Hanqing Pan ${ }^{\dagger}$, Serena Low ${ }^{\dagger}$, Nisala Weerasuriya, Bingli Wang and Young-Seok Shon ${ }^{*}$ (B)
}

\begin{abstract}
This article presents the influence of capping ligand and surface interaction types on the coarsening or reshaping behavior of surface-immobilized gold nanoparticles with different core size and shape. The morphological transformation of gold nanoparticles and nanorods on graphene oxide upon heating at temperatures ranging from 50 to $200{ }^{\circ} \mathrm{C}$ was investigated. The aggregation and coarsening behaviors of spherical nanoparticles on graphene oxide were slightly affected by the core size of nanoparticles $(\sim 1,3$, and $10 \mathrm{~nm})$. The comparison of two different surface ligands revealed that glutathione ligands provide much better protection than cetyltrimethylammonium bromide ligands against the morphological transformation of nanoparticles. In addition, the evaluation of surface binding interactions indicated that the attachment of nanoparticles and nanorods onto graphene oxide with additional thiol functional groups could improve the immobilization of particles and therefore decelerate coarsening and reshaping of nanoparticle and nanorods.
\end{abstract}

Keywords: Nanoparticles, Nanorods, Graphene oxide, Stability, Morphology, Gold

\section{Introduction}

Metal nanoparticles are a class of functional materials with unique physical and chemical properties, which are closely related to their size, shape, composition, and structure [1, 2]. Especially, gold nanoparticles (AuNP) and gold nanorods (AuNR) have gained a lot of attention because of their applications in catalysis, electronics, and sensors [2-11]. They have also exhibited many attractive features such as strong plasmonic activity, facile ligand functionalization, water solubility, and excellent biocompatibility, which have made them good candidates for biomedical applications [6, 7, 9-17]. Deposition of AuNPs on solid supports has often been necessary for various reasons including enhanced stability and synergistic activity $[2,5,10,11,14]$. Therefore, the preparation of nanoparticle hybrids with the appropriate support materials and understanding the influence of supports

\footnotetext{
*Correspondence: ys.shon@csulb.edu

${ }^{\dagger}$ Hanqing Pan and Serena Low contributed equally to this work

Department of Chemistry and Biochemistry, California State University, Long Beach, 1250 Bellflower Blvd, Long Beach, CA 90840, USA
}

to nanoparticles are critical to the expansion of practical applications of AuNPs [18, 19].

Since AuNPs anchored on graphene families could exhibit interesting catalytic, electrical and optical activities $[2,5,8,10,11,20]$, understanding the nature and extent of morphological transformation of gold nanoparticle-graphene oxide (AuNP-GO) hybrids could maximize their potentials for many practical applications [18-23]. Previous studies from our research group have shown that the presence of GO supports negatively affects the stability of AuNPs and AuNRs causing their core to undergo size and/or shape evolutions more easily [24]. The disruption and stripping of the protecting organic ligands by GO through strong electrostatic interactions were proposed as a main mechanism of nanoparticle destabilization on GO.

The gradual changes in size and shape of nanoparticles, when they are exposed to heat or light irradiation $[21,22$, 25-27], could seriously impede their long-term technological advancements for device and catalysis applications. Therefore, further understanding of various factors 
determining morphological transformations of nanoparticles is required for developing new strategies for improving their overall stability and performance. In this study, AuNPs with different core sizes are assembled on the surface of GO to study their heat-induced coarsening behavior. AuNPs with different surface capping ligands (glutathione and cetyltrimethylammonium bromide $(\mathrm{CTAB}))$ on $\mathrm{GO}$ was also prepared to understand the relationship between the stability of AuNPs and the type of surface ligand-nanoparticle interactions. The chemical interaction or bond formation between GO supports and ligand-capped AuNPs was also controlled by using thiol-functionalized $\mathrm{GO}(\mathrm{tGO})$ to see the influence of additional metal-sulfur bond against the morphological transformation of AuNP and AuNR. Various AuNP-GO and AuNR-GO hybrid materials are annealed from 50 to $200{ }^{\circ} \mathrm{C}$ to examine the influence of various structural components.

\section{Experimentals}

Detailed information regarding materials, synthetic procedures, and characterizations $\left({ }^{1} \mathrm{H}\right.$ NMR, FT-IR, and TGA) for glutathione-capped gold nanoparticles (gAuNP) [28-31], cetyltrimethylammonium bromide (CTAB)-capped gold nanoparticles (cAuNP) [9], and CTAB-capped gold nanorods (cAuNR) [9], and instrumental methods used to conduct the research presented in this article are provided in the previous publications and Additional file 1 [15, 24, 29].

\subsection{Preparation of thiol-functionalized graphene oxide ( $\mathrm{GOO})$}

Thiol-functionalized graphene oxide was made by following the published procedure [22, 30,32]. First, $5 \mathrm{~mL}$ of 0.4 M EDC (1-ethyl-3-(3-dimethylaminopropyl)carbodiimide), $5 \mathrm{~mL}$ of $0.1 \mathrm{M}$ NHS (N-hydroxysuccinimide), and $5 \mathrm{~mL} 0.1 \mathrm{M} \mathrm{L}$-cysteine were prepared. A $0.5 \mathrm{~mL}$ solution of concentrated hydrochloric acid was added to the $\mathrm{L}$-cysteine solution to dissolve the L-cysteine crystals. Solutions of EDC, NHS, and L-cysteine were added to $8 \mathrm{~mL}$ of $4 \mathrm{mg} / \mathrm{mL}$ graphene oxide suspension in the order mentioned. As soon as the EDC/NHS/L-cysteine solutions were added, graphene oxide begins to separate from solution due to the decrease in polarity of GO with the incorporation of thiol functional groups from L-cysteine. The mixture is left sitting for $10 \mathrm{~min}$, then centrifuged. The supernatant is discarded, and the precipitate was washed with nanopure water and centrifuged to precipitate the tGO. This process was repeated twice. The final product was left to dry in a vacuum overnight. IR spectra of isolated tGO showed the presence of $\mathrm{S}-\mathrm{H}$ stretching bands at $\sim 2565 \mathrm{~cm}^{-1}$.

\subsection{Preparation of gold nanoparticle-graphene oxide (AuNP-GO) and nanorod-graphene oxide (AuNR-GO) hybrids}

Graphene oxide solution was made by dispersing GO flakes in nanopure water. GO flakes were not readily dispersed in water, hence the mixture was sonicated for 30 min until the $\mathrm{GO}$ formed a homogeneous dispersion. The concentration of GO solution was $1 \mathrm{mg} / \mathrm{mL}$. The GO dispersion in water exhibited long-term stability. The gAuNP of size $\sim 1,3$, and $10 \mathrm{~nm}$, cAuNP, and cAuNR were bonded to GO as follows: Gold nanorods/nanoparticles were dissolved in water $(\sim 1 \mathrm{mg} / \mathrm{mL})$ forming homogeneous solutions. The solutions of GO and gold nanorods/nanoparticles were mixed at a $1: 1$ ratio $(\mathrm{v} / \mathrm{v}$ : $1 \mathrm{~mL}$ GO solution to $1 \mathrm{~mL}$ gold nanorod/nanoparticle solution) for $24 \mathrm{~h}$.

To prepare the hybrids of tGO with AuNP and AuNR, the dried tGO was re-dispersed in nanopure water and the aqueous solutions of AuNP and AuNR were added to this solution. The mixture was then stirred at room temperature for $24 \mathrm{~h}$ to allow the formation of metal-sulfur bonds, so that the nanoparticles and nanorods can be covalently anchored to graphene oxide. The final hybrid product is then centrifuged, and the supernatant was discarded. The resulting precipitate was stored in a vacuum desiccator for at least $72 \mathrm{~h}$ to remove all solvents.

\subsection{Heat-treatment of AuNP-GO and AuNR-GO hybrids}

The hybrids were heated from 50 to $200{ }^{\circ} \mathrm{C}$, at $50{ }^{\circ} \mathrm{C}$ increments using a Barnstead Thermolyne 1300 Furnace. Each sample was heated for $1 \mathrm{~h}$ in a glass vial in air. Solvents were removed to dryness before the particle samples were heated in powder form.

\section{Results and discussion}

\subsection{Gold nanoparticles with different sizes and stabilizing ligands}

UV-vis spectra of gAuNP with different core sizes are shown in Fig. 1a, which confirmed the optical properties of gold nanoparticles corresponding to their expected sizes. The UV-vis spectrum of gAuNP ${ }_{10 \mathrm{~nm}}$ exhibits strong surface plasmon (SP) bands at $\sim 520 \mathrm{~nm}$, while that of $\mathrm{gAuNP}_{3 \mathrm{~nm}}$ show less prominent SP bands at the similar wavelength [29]. The UV-vis spectrum of small and monodispersed gAuNP ${ }_{1 \mathrm{~nm}}$ does not possess plasmon bands, but have three absorption bands at 680, 450, and $410 \mathrm{~nm}$, which are in a good agreement with previous reports published on the characteristics of $\mathrm{Au}_{25}(\mathrm{GS})_{18}$ $[30,31]$. Both cAuNP 3 nm and cAuNR were prepared following a published method by Castellana et al. [9]. The UV-vis spectrum of $\mathrm{cAuNP}_{3 \mathrm{~nm}}$ showing small SP bands at $\sim 520 \mathrm{~nm}$ is quite similar to that of gAuNP $3 \mathrm{~nm}$ (Fig. 1b). 

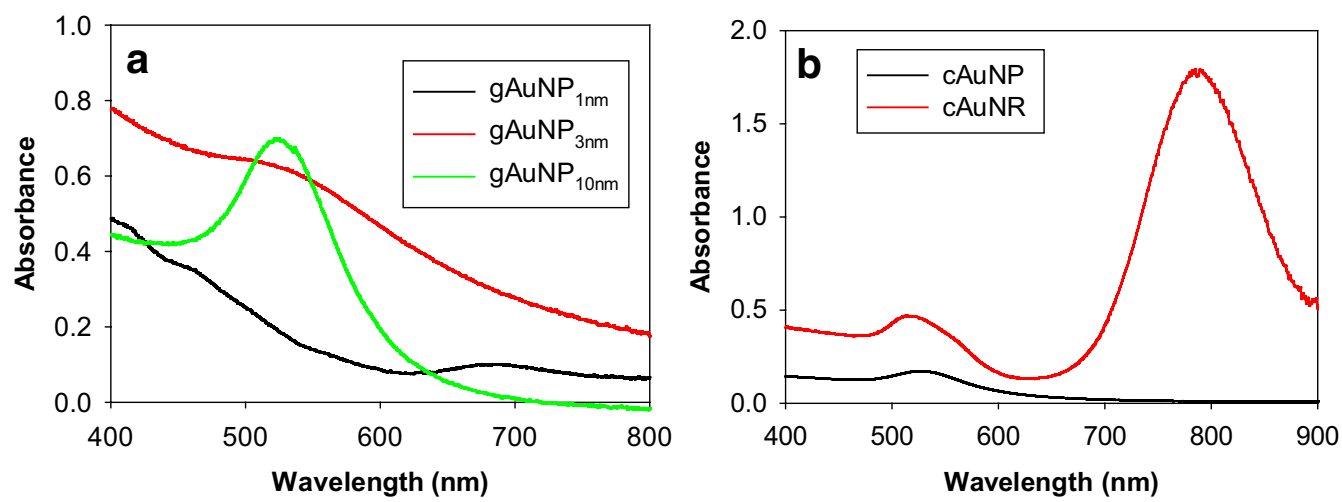

Fig. 1 UV-vis spectra of a gAuNP with core sizes of $\sim 1,3$, and $10 \mathrm{~nm}$ and $\mathbf{b}$ cAuNP and cAuNR

The characteristic plasmon bands at $520 \mathrm{~nm}$ (transverse) and $780 \mathrm{~nm}$ (longitudinal) shown in the UV-vis spectrum of cAuNR confirmed the successful preparation of monodisperse gold nanorods.

TEM images and their respective histograms showing size distribution of gAuNP are shown in Fig. 2. TEM images of both gAuNP ${ }_{10 \mathrm{~nm}}$ and gAuNP 3 nm (Fig. 2a, b), respectively) show that the nanoparticles are monodispersed and not aggregated. Histograms show that the majority of $\mathrm{gAuNP}_{3 \mathrm{~nm}}$ are indeed populated around $\sim 3 \mathrm{~nm}$ and the gAuNP ${ }_{10 \mathrm{~nm}}$ have an average size of $\sim 11.5 \mathrm{~nm}$. TEM image (Fig. 2c) of the small and monodispersed gAuNP $_{1 \mathrm{~nm}}$ corresponds well with UV-vis results, being that they are too small to possess plasmon bands. The histogram of gAuNP ${ }_{1 \mathrm{~nm}}$ shows that the core size of the nanoparticles is highly populated at $\sim 1.2 \mathrm{~nm}$.

TEM images of cAuNP 3 nm and cAuNR (Fig. 3) show that the $\mathrm{cAuNP}_{3 \mathrm{~nm}}$ have spherical shape with average core size of $\sim 2.7 \mathrm{~nm}$ and the cAuNR are highly ordered, uniform in size and shape, and show no evidence of coarsening. Histograms for cAuNR show size distributions for both length and width with the majority of nanorods being $\sim 55 \mathrm{~nm}$ in length and $15 \mathrm{~nm}$ in width (an aspect ratio of 3.5:1). The first column of Table 1 summarizes the average core size and their respective standard deviations of the gAuNP, cAuNP, and cAuNR.

\subsection{Gold nanoparticles and nanorods on graphene oxide}

The gAuNP with different sizes were attached to the surface of GO via non-covalent interactions such as hydrogen bonding and electrostatic interactions between $\mathrm{COOH} / \mathrm{OH}$ groups of $\mathrm{GO}$ and $\mathrm{COO}^{-} / \mathrm{NH}_{2}$ groups of glutathione ligands on gAuNP. The cAuNP and cAuNR were self-assembled onto the surface of GO mostly via electrostatic interactions of $\mathrm{R}_{4} \mathrm{~N}^{+}$groups of bilayer CTAB ligands with the $\mathrm{COO}^{-}$groups of GO. UV-vis spectra of gAuNP-GO with different core sizes, $\mathrm{cAuNP}_{3 \mathrm{~nm}}-\mathrm{GO}$, and
cAuNR-GO are shown in Fig. 4. When gAuNP ${ }_{10 \mathrm{~nm}}$ are assembled onto GO, the SP bands of gold broadens and red-shifts, which are consistent with the results reported by Huang et al. [33]. It has been shown that such a change in the plasmon band of the large gold nanoparticles in the GO hybrids indicate the significant formation of AuNP aggregates on the GO sheets [34, 35]. After the adsorption of gAuNP $3 \mathrm{~nm}$ on GO, the SP bands of gold at $\sim 520 \mathrm{~nm}$ mostly disappear as shown in the UV-vis spectrum. In our previous work, we found that this optical change is not associated with the core size change of AuNP after adsorption [24]. For the UV-vis spectrum of $\mathrm{gAuNP}_{1 \mathrm{~nm}}$, three characteristic absorption bands at 680,450 , and $410 \mathrm{~nm}$ completely disappeared after the assembly onto GO exhibiting only an exponential decay in absorbance with a decrease in energy. The spectra feature now resembles that of 1.5-2 nm AuNP which do not possess any significant adsorption and SP bands in the UV-vis spectra. Recently, Ghosh et al. [36] have shown that $\mathrm{Au}_{25} \mathrm{SR}_{18}$ clusters undergo core size evolution to $\mathrm{Au}_{135}$ clusters after the deposition on GO in THF. This result indicated that the disappearance of three absorption bands after adsorption onto GO and an appearance of small hints of SP bands at $520 \mathrm{~nm}$ in UV-vis spectrum of the gAuNP $1 \mathrm{~nm}$-GO hybrids was likely caused by the core size evolution of small and less stable gold cluster core to gold nanoparticles with core size of $>1.4 \mathrm{~nm}$.

When $\mathrm{cAuNP}_{3 \mathrm{~nm}}$ and cAuNR are deposited on GO, the SP bands at $\sim 520 \mathrm{~nm}$ almost completely disappear and the longitudinal plasmon band of cAuNR decreases in intensity (Fig. 4b), which is another piece of evidence for electronic and optical interference of GO for plasmonic nanostructures. Such changes in the plasmon response of cAuNR adsorbed on GO have also been observed by others $[37,38]$. The absence of any shift for the longitudinal plasmon band of cAuNR indicated, however, that the aspect ratio of cAuNR mostly remained the same. 

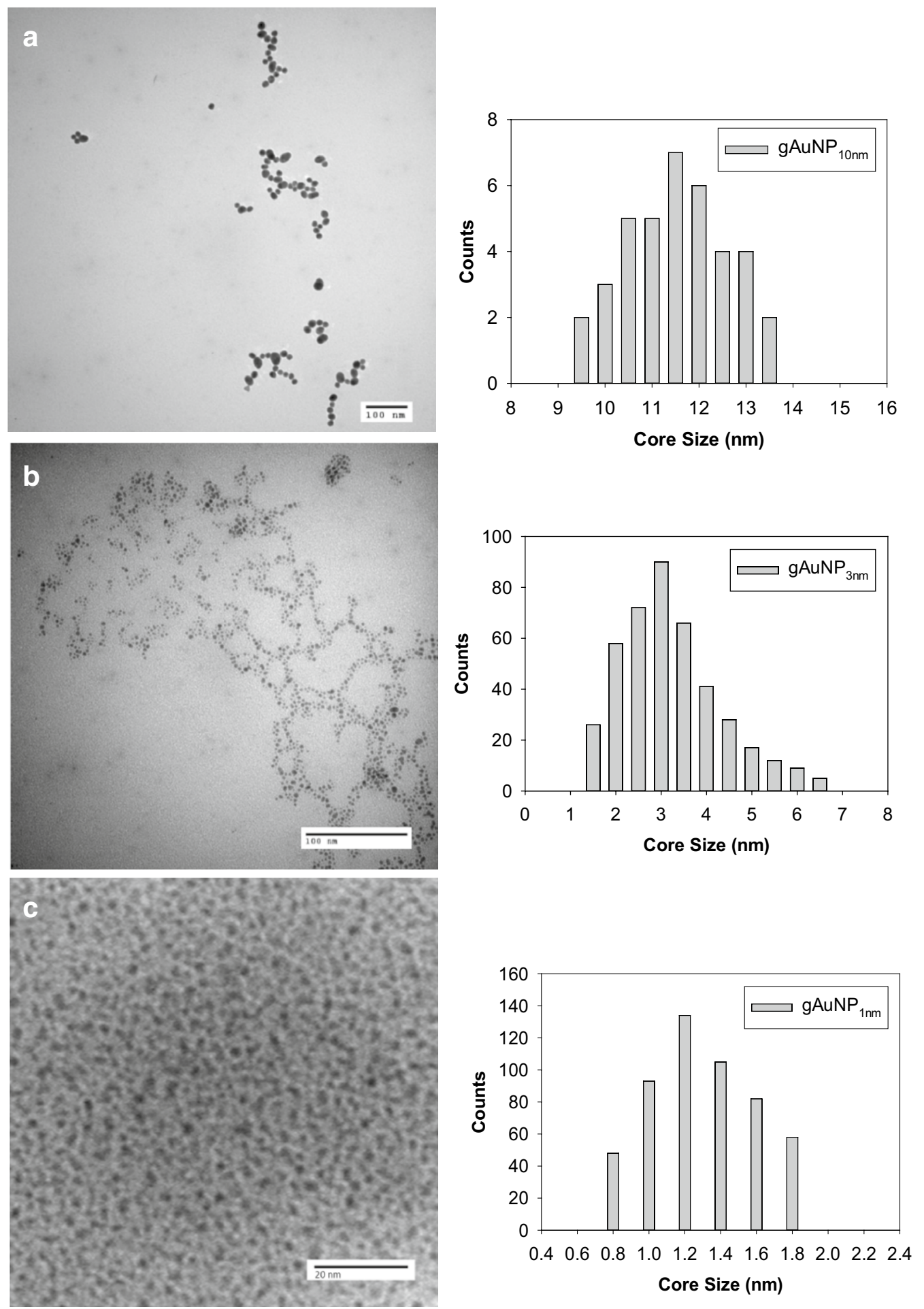

Fig. 2 TEM images and histograms of a gAuNP $10 \mathrm{~nm}, \mathbf{b} g A u N P_{3 \mathrm{~nm}}$, and $\mathbf{c} g A u N P_{1 \mathrm{~nm}}$

Additional file 1: Figure S1 shows TEM images of various gAuNP-GO hybrid materials and their respective histograms showing size distribution. GO sheets are apparent in all the TEM images as darker regions. The actual particle core size of gAuNP $10 \mathrm{~nm}-\mathrm{GO}$ is estimated to be $\sim 10 \mathrm{~nm}$, which is similar to the size of free standing 

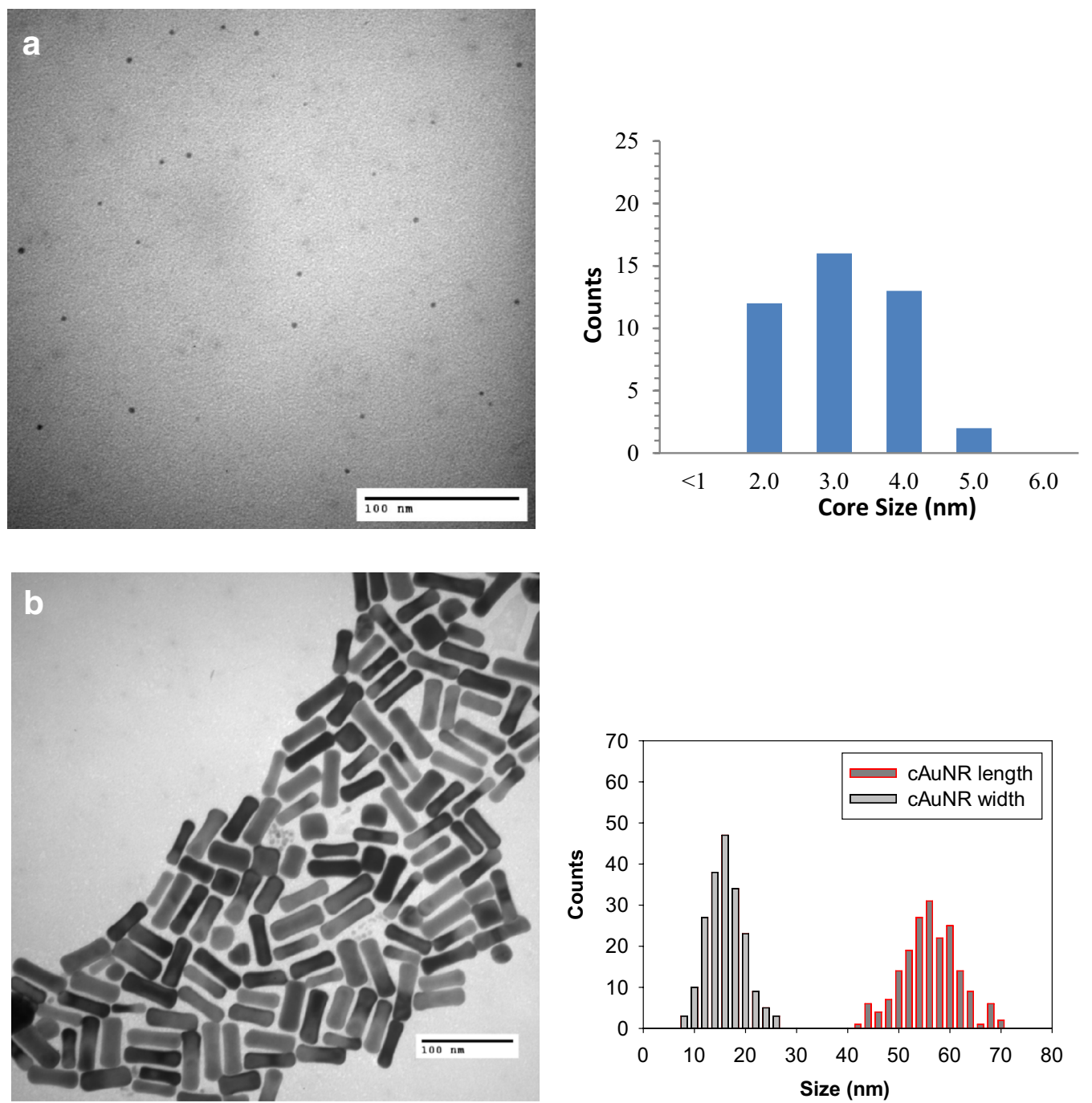

Fig. 3 TEM images and histograms of $\mathbf{a} C A u N P_{3 n m}$ and $\mathbf{b} C A U N R$

Table 1 Core size evolution of gold nanoparticles and nanorods (nanometers)

\begin{tabular}{|c|c|c|c|c|c|c|}
\hline & Free standing & $\begin{array}{l}\text { Supported on GO } \\
\text { (or tGO) }\end{array}$ & Heated at $50^{\circ} \mathrm{C}$ & Heated at $100^{\circ} \mathrm{C}$ & Heated at $150^{\circ} \mathrm{C}$ & Heated at $200^{\circ} \mathrm{C}$ \\
\hline $\mathrm{gAuNP}_{10 \mathrm{~nm}}$ & $11.1 \pm 1.2$ & $10.1 \pm 6.9$ & $10.9 \pm 4.8$ & $11.5 \pm 6.6$ & $20.2 \pm 8.6$ & $22.1 \pm 6.2$ \\
\hline $\mathrm{gAuNP}_{3 \mathrm{~nm}}$ & $3.0 \pm 1.4$ & $\begin{array}{l}3.0 \pm 0.9^{\mathrm{a}} \\
2.9 \pm 1.1 \\
(\mathrm{on} \mathrm{tGO})\end{array}$ & $\begin{array}{l}3.1 \pm 1.8^{\mathrm{a}} \\
3.1 \pm 1.1 \\
(\mathrm{on} \mathrm{tGO})\end{array}$ & $\begin{array}{l}3.3 \pm 1.6^{a} \\
3.3 \pm 1.6 \\
(\text { on } \mathrm{tGO})\end{array}$ & $\begin{array}{l}7.0 \pm 3.6^{\mathrm{a}} \\
5.1 \pm 2.0 \\
\text { (on } \mathrm{tGO} \text { ) }\end{array}$ & $\begin{array}{l}10.1 \pm 5.4^{\mathrm{a}} \\
7.6 \pm 3.8 \\
\text { (on } \mathrm{GO} \text { ) }\end{array}$ \\
\hline $\mathrm{gAuNP}_{1 \mathrm{~nm}}$ & $1.2 \pm 0.3$ & $1.3 \pm 0.4$ & $1.4 \pm 0.5$ & $1.4 \pm 0.7$ & $4.7 \pm 0.8$ & $5.3 \pm 1.6$ \\
\hline $\mathrm{CAuNP}_{3 \mathrm{~nm}}$ & $2.7 \pm 1.1$ & $3.6 \pm 1.6$ & $10.0 \pm 6.0$ & $13.7 \pm 10.5$ & $68.5 \pm 20.1$ & - \\
\hline cAuNR & $\begin{array}{l}53.6 \pm 8.2 \\
15.1 \pm 3.6\end{array}$ & $\begin{array}{l}53.8 \pm 13.2^{\mathrm{a}} \\
17.3 \pm 6.3^{\mathrm{a}} \\
54.4 \pm 7.1 \\
14.8 \pm 2.3 \\
\text { (on tGO) }\end{array}$ & $\begin{array}{l}89.4 \pm 52.3^{a} \\
\text { n.a } a^{\mathrm{a}} \\
52.4 \pm 6.7 \\
12.9 \pm 2.2 \\
\text { (on } \mathrm{tGO} \text { ) }\end{array}$ & $\begin{array}{l}- \\
-\end{array}$ & $\begin{array}{l}- \\
-\end{array}$ & - \\
\hline
\end{tabular}

a The data are from the previous publication [24] 

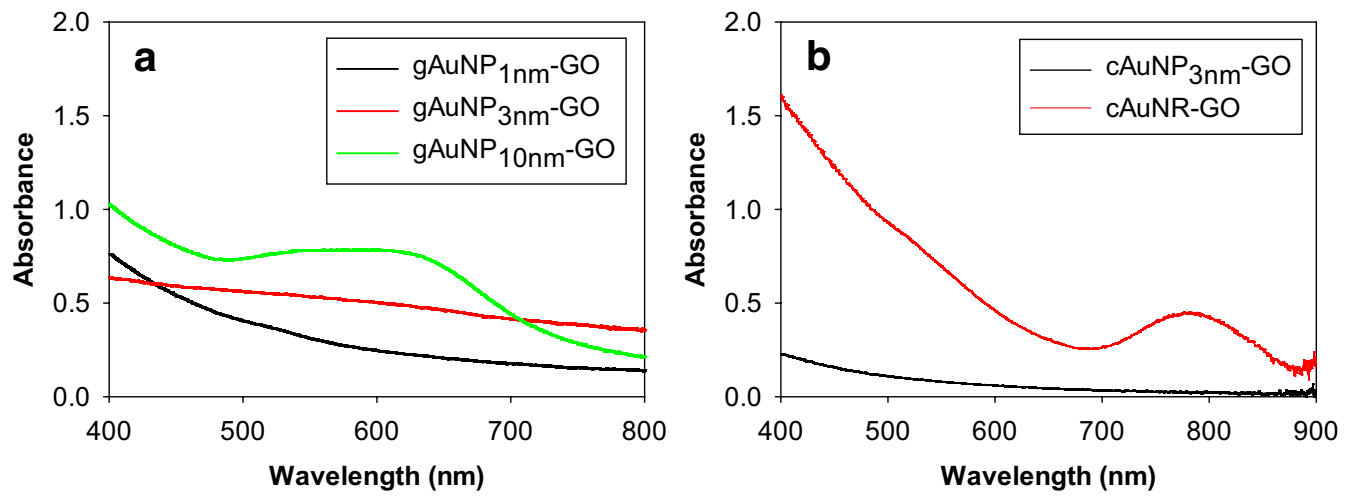

Fig. 4 UV-vis spectra of a gAuNP ${ }_{10 n m}-G O, g A_{u N P} P_{3 n m}-G O$, and gAuNP ${ }_{1 n m}-G O$ and $\mathbf{b} c A u N P_{3 n m}-G O$ and $c A u N R-G O$

gAuNP $_{10 \mathrm{~nm}}$ (Table 1). However, the images showed many particles to be positioned closely together making an accurate histogram analysis a bit more difficult. These apparent formations of nanoparticle aggregates must be the main reason for the large red shift of the SP bands of gAuNP $10 \mathrm{~nm}$ after GO adsorption. TEM image and histogram results agreed that the $\mathrm{gAuNP}_{3 \mathrm{~nm}}-\mathrm{GO}$ have relatively well dispersed nanoparticles with the average core size near $\sim 3 \mathrm{~nm}$. The histogram results indicated gAuNP $3 \mathrm{~nm}$ are relatively stable after GO immobilization showing no evidence of core size evolution. For gAuNP ${ }_{1 \mathrm{~nm}}$-GO hybrids, TEM images and histogram results indicated there is a slight increase in average core size of gAuNP $1 \mathrm{~nm}$ after adsorption onto GO. This size change corresponded well with the UV-vis results of gAuNP $1 \mathrm{~nm}^{-\mathrm{GO}}$ and support the previous discovery reported by Ghosh et al. [36] regarding the size evolution of $\mathrm{Au}_{25}$ clusters when in contact with graphene substrate.

TEM results shown in Additional file 1: Figure S2 indicated that $\mathrm{cAuNP}_{3 \mathrm{~nm}}$ and cAuNR are immobilized on GO without any aggregation or reshaping maintaining a similar core size and an aspect ratio of over $3(\sim 54 \mathrm{~nm}$ in length and $\sim 17 \mathrm{~nm}$ in width), respectively. The TEM results agreed relatively well with their corresponding UV-vis spectra of their original sizes and shapes shown in Fig. 4b. The dampening of the plasmon bands of $\mathrm{cAuNP}_{3 \mathrm{~nm}}$ and cAuNR at $\sim 520 \mathrm{~nm}$ was clearly not a result of any significant morphological size or shape changes.

When comparing the core sizes of the nanoparticles before and after their assembly onto GO, it is clear that the use of pre-formed nanoparticles mostly affords the retention of core size and dispersity of assembled nanoparticles without any significant change. However, very small clusters $(\sim 1 \mathrm{~nm})$ and large colloidal nanoparticles $(>10 \mathrm{~nm})$ that usually exhibit low stability alone in solution phase are also susceptible to a slight change in their morphologies after immobilization on GO. This result is confirmed by TEM analysis and the changes in their optical activity. Gold nanorods immobilized on GO avoided extensive aggregations suggesting the presence of CTAB bilayer ligands on their surfaces.

\subsection{Effects of core size on coarsening of gAuNP on GO}

Our previous work has shown that the presence of GO facilitates the coarsening of gAuNP ${ }_{3 n m}$ [24]. As shown in Table 1, large increases in the average core size of $\mathrm{gAuNP}_{3 \mathrm{~nm}}$ on $\mathrm{GO}$ took place at heating temperatures between 100 and $150{ }^{\circ} \mathrm{C}$. The transition, however, took place between 150 and $200{ }^{\circ} \mathrm{C}$ for free standing gAuNP $_{3 \mathrm{~nm}}$ [24]. The hypothesis was that the GO, being a flexible material, can bend and wrap around the nanoparticles and chemically interact with the ligands due to the presence of many oxygen-containing functional groups. The ligands on AuNP are then pulled away from the nanoparticle and spilled over to GO during heat treatment facilitating the coarsening of the nanoparticles.

Heat treatments of gAuNP ${ }_{10 \mathrm{~nm}^{-}}-\mathrm{GO}$ and gAuNP ${ }_{1 \mathrm{~nm}}-\mathrm{GO}$ were performed from 50 to $200{ }^{\circ} \mathrm{C}$ at $50{ }^{\circ} \mathrm{C}$ increments to see the effects of particle size on the heat-induced coarsening behavior of gAuNP. UV-vis spectra of $\mathrm{gAuNP}_{10 \mathrm{~nm}}-\mathrm{GO}$ showed the decreased intensity and significant broadening of SP bands after the heat treatments of the hybrids at $100^{\circ} \mathrm{C}$, which is lower than the temperature required for $\mathrm{gAuNP}_{3 \mathrm{~nm}}$-GO (Additional file 1: Figure S3). The transitions continued with heating at increased temperatures and quickly reached the status of film-like insoluble materials showing only small scattering effects [39-41]. This fast transition would likely be caused by the formation of particle aggregations upon the adsorption of gAuNP $_{10 \mathrm{~nm}}$ on GO.

TEM results clearly indicated that the gAuNP ${ }_{10 \mathrm{~nm}}$ on $\mathrm{GO}$ undergo coarsening at the heating temperature at or above $100{ }^{\circ} \mathrm{C}$ (Fig. 5). After heat treatment at $100{ }^{\circ} \mathrm{C}$ 


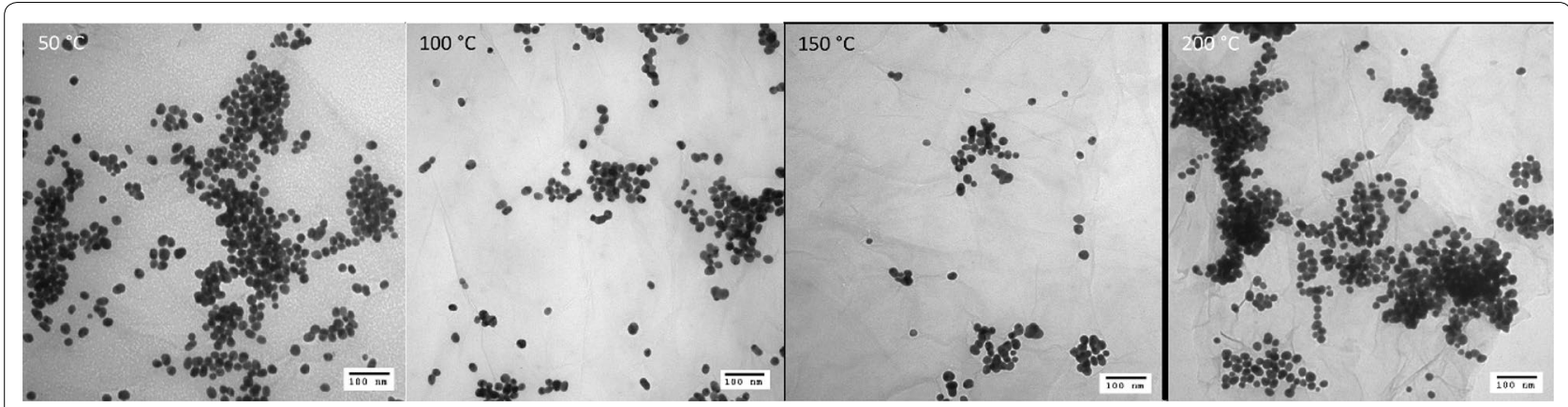

Fig. 5 TEM images (scale bars $=100 \mathrm{~nm}$ ) and size distribution of gAuNP $10 \mathrm{~nm}-\mathrm{GO}$ heated at 50, 100, 150, and $200^{\circ} \mathrm{C}$

(Fig. $8,100{ }^{\circ} \mathrm{C}$ ), particles are more aggregated with a few particles slightly merging together. After the heat treatment at 150 and $200{ }^{\circ} \mathrm{C}$ (Fig. $8,150{ }^{\circ} \mathrm{C}, 200{ }^{\circ} \mathrm{C}$ ), it is clearly visible from TEM images that the particles are coarsening. Table 1 summarizes the changes in the particle size of the heated gAuNP $10 \mathrm{~nm}$ on GO. A large increase in the average core size of $\mathrm{gAuNP}_{10 \mathrm{~nm}}$ was observed from 100 to $150{ }^{\circ} \mathrm{C}$ suggesting the overall transitions are similar to that of $\mathrm{gAuNP}_{3 \mathrm{~nm}}$ on $\mathrm{GO}$ and relatively in good agreement with both UV-vis results shown in Additional file 1: Figure S3 and those of gAuNP $3 \mathrm{~nm}$-GO hybrids shown previously (Table 1).

UV-vis spectra of gAuNP ${ }_{1 \mathrm{~nm}}$-GO (Additional file 1: Figure S4) show that the transition is gradually taking place throughout the heating temperature between 50 and $200{ }^{\circ} \mathrm{C}$ with decreasing intensity of spectra. The film-like spectral feature has appeared after heat treatments at $200{ }^{\circ} \mathrm{C}$. This overall transition after heat treatment resembles quite similarly to that of gAuNP $_{10 \mathrm{~nm}}-\mathrm{GO}$ as shown above. The presence of small SP bands of gold is apparent for gAuNP $1 \mathrm{~nm}^{-G O}$ after heating at $50{ }^{\circ} \mathrm{C}$, which indicates some aggregation and coarsening of nanoparticles to slightly larger nanoparticles $(\sim 1.5-2.5 \mathrm{~nm})$. The absence of any strong SP bands after heating at high temperature, however, indicates that the significant coarsening of gAuNP $1 \mathrm{~nm}$ on GO to larger $(>3 \mathrm{~nm})$ particles did not take place.

TEM images of the heated gAuNP ${ }_{1 \mathrm{~nm}}-\mathrm{GO}$ are shown in Additional file 1: Figure S5 and the changes in the average core size and distribution are summarized in Table 1. The results suggested that the more rapid transition is observed at temperature below $150{ }^{\circ} \mathrm{C}$ for the $\mathrm{gAuNP}_{1 \mathrm{~nm}}-\mathrm{GO}$ compared to other gAuNP on GO. The gAuNP ${ }_{1 \mathrm{~nm}}-\mathrm{GO}$ began to reach the extensive coarsening stage and became mostly insoluble during the heat treatment at $200{ }^{\circ} \mathrm{C}$. These results also corresponded well with the data obtained from UV-vis spectra.

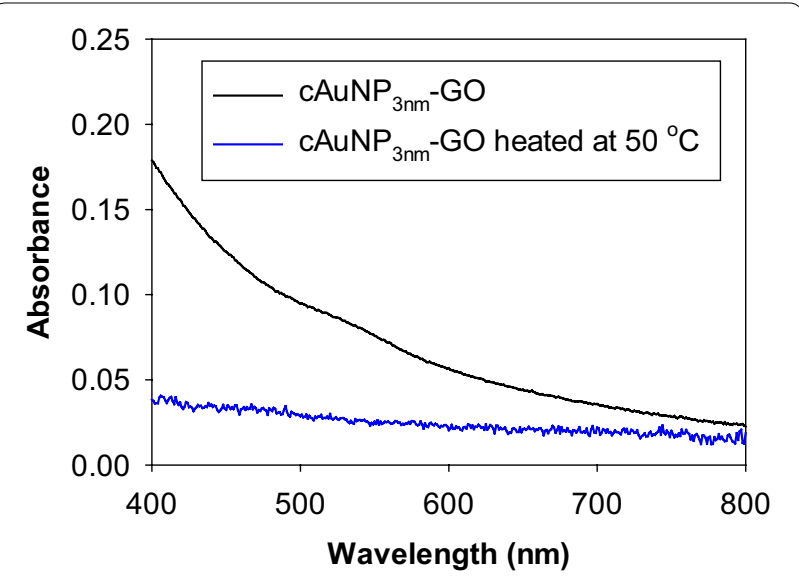

Fig. 6 UV-vis spectra of $\mathrm{CAuNP}_{3 \mathrm{~nm}}$-GO before and after heat treatment at $50^{\circ} \mathrm{C}$

\subsection{Effects of capping ligands for coarsening of gold nanoparticles on graphene oxide $\left(\mathrm{gAuNP}_{3 \mathrm{~nm}}-\mathrm{GO}\right.$ vs cAuNP ${ }_{3 \mathrm{~nm}}-\mathrm{GO}$ )}

To understand the effects of capping ligands on the stability of assembled gold nanoparticles, the heat treatment of $\mathrm{cAuNP}_{3 \mathrm{~nm}}-\mathrm{GO}$ was performed and its results were compared to those of $\mathrm{gAuNP}_{3 \mathrm{~nm}}-\mathrm{GO}$ published in our previous report [24]. UV-vis spectra of the heated $\mathrm{cAuNP}_{3 \mathrm{~nm}}-\mathrm{GO}$ were somewhat different to those of gAuNP $_{3 \mathrm{~nm}}-\mathrm{GO}$ showing a progressive depression of absorbance intensity. The UV-vis spectra of heattreated $\mathrm{cAuNP}_{3 \mathrm{~nm}}-\mathrm{GO}$ would only reveal the rapid decrease in the overall absorbance without the appearance of SP or even scattering bands (Fig. 6). This is due to the rapid flocculation of hybrid materials even at $50{ }^{\circ} \mathrm{C}$ causing the visible precipitation of $\mathrm{cAuNP}_{3 \mathrm{~nm}}$ $-\mathrm{GO}$. TEM images of heated $\mathrm{cAuNP}_{3 \mathrm{~nm}}-\mathrm{GO}$ clearly indicated that cAuNP undergo coarsening to larger particles when heated at $50{ }^{\circ} \mathrm{C}$ (Fig. 7). The histogram analyses results shown in Fig. 7 and Table 1 confirmed 

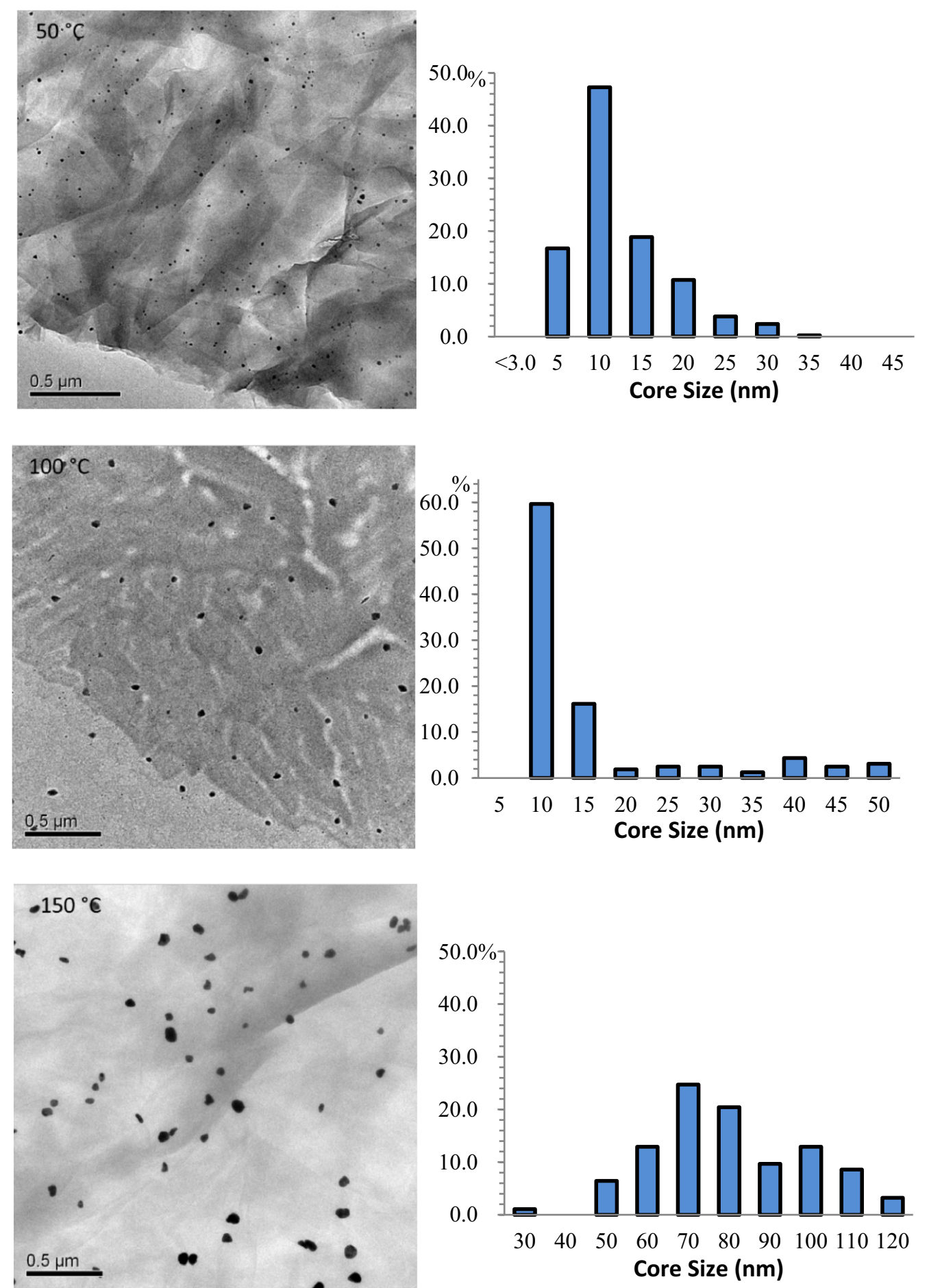

Fig. 7 TEM images and histograms of $\mathrm{CAuNP}_{3 \mathrm{~nm}}-\mathrm{GO}$ heated at 50,100 , and $150^{\circ} \mathrm{C}$

a very fast increase in the average size of cAuNP to $\sim 10,14$, and $68 \mathrm{~nm}$ after heat treatments at $50{ }^{\circ} \mathrm{C}$, $100{ }^{\circ} \mathrm{C}$, and $150{ }^{\circ} \mathrm{C}$, respectively. The large increases in average size of cAuNP after heat treatment suggested that the extensive coarsening of cAuNP on GO takes place at lower temperature. The weak binding affinity of CTAB with AuNP most likely causes CTAB to be 


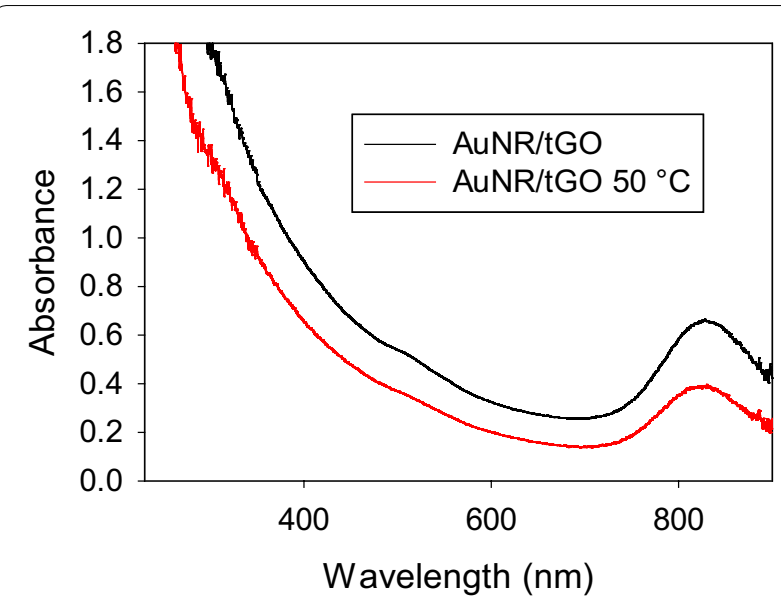

Fig. 8 UV-vis spectra of $C A u N R-t G O$ before and after heat treatment at $50^{\circ} \mathrm{C}$

more easily stripped by the GO and AuNP to diffuse on $\mathrm{GO}$ to form aggregates at faster rate.

\subsection{Effects of additional thiol functional groups on graphene oxide for coarsening of gold nanoparticles and nanorods (GO vs tGO)}

The gAuNP 3 nm and cAuNR were also adsorbed on thiolfunctionalized GO (tGO) to study the effect of additional thiol-gold interaction between nanoparticles and GO during coarsening or reshaping. The tGO was prepared by the well-established method using the coupling of L-cysteine to graphene oxide in the presence of EDC and NHS [22, 30,32]. The tGO was then mixed with a solution containing gAuNP 3 nm or cAuNR which resulted in the self-assembly of nanoparticles onto the surface of tGO with the formation of gold-sulfur bonds in addition to all electrostatic interactions unmodified GO provide. TEM and histogram results showed that the gAuNP $_{3 \mathrm{~nm}^{-}}$-tGO still have the surface immobilized particles with the average core size near $\sim 3 \mathrm{~nm}$ (Additional file 1: Figure S6). Any immediate aggregation after the adsorption of gAuNP $3 \mathrm{~nm}$ on tGO was not observed from TEM results.

Additional file 1: Figure $\mathrm{S} 7$ shows the UV-vis spectra

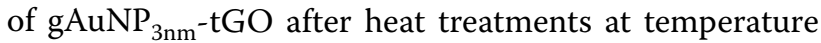
ranging from 50 to $200{ }^{\circ} \mathrm{C}$. The intensity of SP bands along with the overall absorbance spectra, which show an exponential decay from lower wavelength to higher wavelength, continuously decreased after heat treatments at the range between 50 and $150^{\circ} \mathrm{C}$. The results were almost identical with the ones observed for gAuNP $_{3 \mathrm{~nm}}-\mathrm{GO}$ indicating the particles have begun to aggregate and become partially insoluble [24]. The UV-vis spectra of gAuNP $_{3 \mathrm{~nm}}$-tGO heated at $200{ }^{\circ} \mathrm{C}$ showed completely different spectral features for the particles heated at high temperature, which resemble the characteristic of localized metallic film-like structures [24, 39-42].

Additional file 1: Figure S8 shows TEM images of heated $\mathrm{gAuNP}_{3 \mathrm{~nm}}-\mathrm{tGO}$ at temperature from 50 to $150{ }^{\circ} \mathrm{C}$. These results showed that the average core size increase occurred the most between 100 and $150{ }^{\circ} \mathrm{C}$ as we have seen from the heat treatments of gAuNP $_{3 \mathrm{~nm}}$-GO. However, after heating at $150{ }^{\circ} \mathrm{C}$, TEM image and histogram results showed that the gAuNP $3 \mathrm{~nm}^{-\mathrm{tGO}}$ had a smaller average core size $(5.09 \pm 1.97 \mathrm{~nm})$ than gAuNP $3 \mathrm{~nm}-\mathrm{GO}(7.04 \pm 3.61 \mathrm{~nm})$. Although the difference is still within the standard deviation of each sample, the result suggests that there might be some stabilizing influence of tGO with the formation of $\mathrm{Au}-\mathrm{S}$ bonds between $\mathrm{tGO}$ and gAuNP (Table 1). The presence of stronger $\mathrm{Au}-\mathrm{S}$ bonds in addition to other electrostatic interactions would provide more robust interactions between nanoparticles and GO. Thus, with the use of tGO, the immobilized AuNP would be more kinetically trapped and become slightly less susceptible to aggregation and coarsening.

The influence of thiol functional group of $\mathrm{tGO}$ on reshaping behavior of cAuNR was also investigated. The cAuNR was adsorbed on the surface of $\mathrm{tGO}$ and the heat treatment results at $50{ }^{\circ} \mathrm{C}$ were compared to those of cAuNR-GO. Figure 8 shows the UV-vis spectrum of cAuNR-tGO which has the longitudinal SP bands of cAuNR-tGO appear at higher wavelength of $\sim 830 \mathrm{~nm}$ compared to those of cAuNR and cAuNR-GO. This shift is likely a result from the plasmonic shift caused by the formation of $\mathrm{Au}-\mathrm{S}$ bonds [6]. TEM image of cAuNRtGO shown in Fig. 9 confirmed the size and shape of cAuNR remained mostly intact after the adsorption on tGO. UV-vis spectra and TEM image of cAuNR-tGO after heat treatment at $50{ }^{\circ} \mathrm{C}$ are also shown in Figs. 8 and 9, respectively. Although the intensity of the longitudinal plasmon band has decreased slightly, the overall spectral feature remains almost constant after heat treatment. This result was different from the previous studies on cAuNR-GO, in which the UV-vis spectra of heated samples at $50{ }^{\circ} \mathrm{C}$ showed a complete disappearance of the plasmon band at $780 \mathrm{~nm}$ [24]. TEM images also showed that there is little change in nanorod shape when cAuNR-tGO is heated at $50{ }^{\circ} \mathrm{C}$. In comparison, cAuNR-GO underwent a complete reshaping of nanorods to spherical particles after the heat treatment at $50{ }^{\circ} \mathrm{C}$ (Table 1) [24]. The results confirmed the presence of stronger interactions between GO and cAuNR and demonstrated dramatically improved stability of AuNR during heat treatments. The heat treatments at temperature $100{ }^{\circ} \mathrm{C}$ or higher, however, caused the 

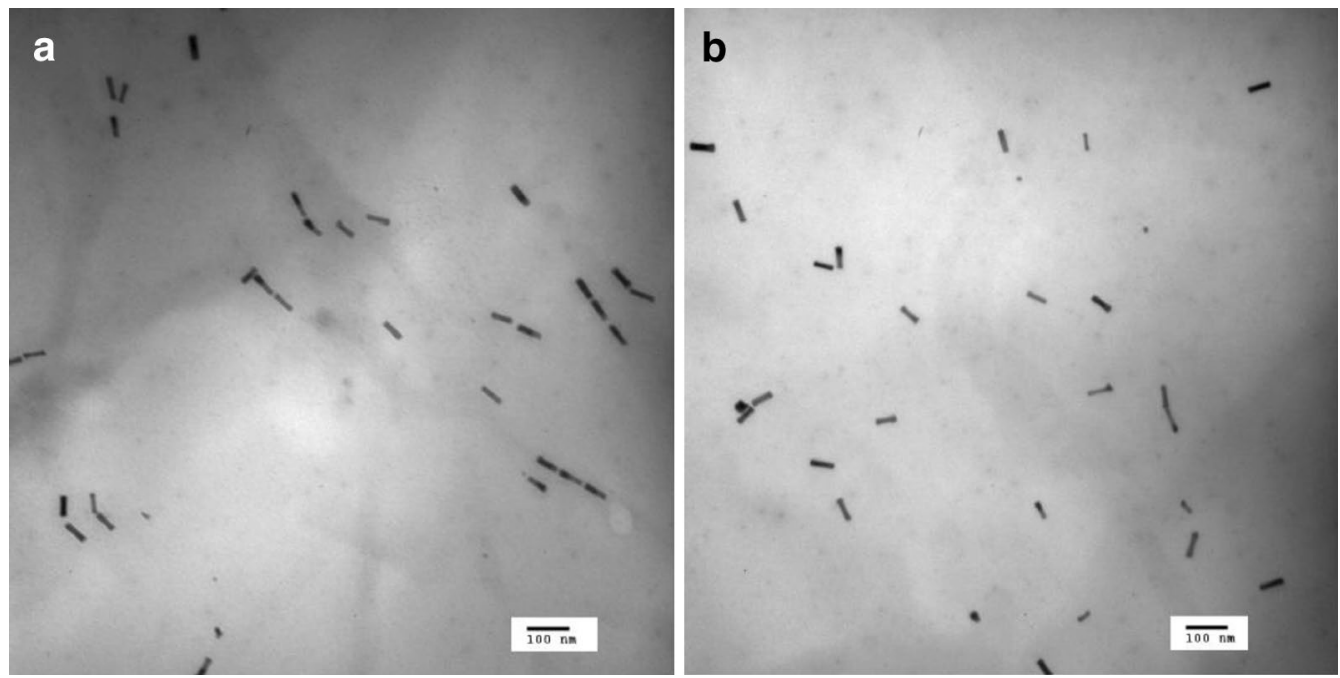

Fig. 9 TEM images (scale bars $=100 \mathrm{~nm}$ ) of $\mathbf{a} c A u N R-t G O$ and $\mathbf{b} c A u N R-t G O$ heated at $50^{\circ} \mathrm{C}$

cAuNR to undergo a complete reshaping to spherical particles.

\section{Conclusions}

In this study, the coarsening behavior of gold nanoparticles on graphene oxide was investigated with the focus on the influence of particle core size, stabilizing ligands, and thiol linkers. For gold nanoparticles, the core size evolution was slightly affected by the size of nanoparticles. Gold nanoparticles smaller than $1.5 \mathrm{~nm}$ and large colloidal gold nanoparticles of $\sim 10 \mathrm{~nm}$ were less stable than gold nanoparticles with $\sim 3 \mathrm{~nm}$ core, undergoing immediate coarsening and/or aggregation upon adsorption on the surface of graphene oxide. The core size evolution during heat treatments at the temperature ranging from 50 to $200{ }^{\circ} \mathrm{C}$, however, was similar for all three gold nanoparticles with different core sizes. Glutathione was observed to be a more stabilizing ligand than CTAB by having a stronger bond with the gold surface and a greater resistance to being removed from the nanoparticles by graphene oxide. Much greater core size evolution was observed from CTAB-capped gold nanoparticles on graphene oxide. The presence of thiol linkers on graphene oxide allowed for the formation of strong $\mathrm{Au}-\mathrm{S}$ bonds that immobilized the nanoparticles strongly onto graphene oxide. The improvement in the stability of nanoparticles by $\mathrm{Au}-\mathrm{S}$ bond formation was even more profound for the gold nanorods. The heat treatments of gold nanorods on graphene oxide at $50{ }^{\circ} \mathrm{C}$ resulted in the complete reshaping of nanorods to spherical particles. Under the same condition, the reshaping of gold nanorods on thiol-functionalized graphene oxide was not observed indicating that the formation of $\mathrm{Au}-\mathrm{S}$ bonds allows the gold nanorod to withstand the thermal energy even after the disruption of CTAB ligands around nanorods. The overall results proved that small modification in the nanoparticle and/or graphene oxide functional groups would have a large influence on intrinsic stability of nanomaterials.

\section{Additional file}

Additional file 1. Materials, synthetic methods, instrumentation methods, and TEM images and UV-Vis spectra of Au nanoparticles and nanorods.

\section{Abbreviations}

AuNP: gold nanoparticles; AuNR: gold nanorods; GO: graphene oxide; CTAB: cetyltrimethylammonium bromide; $\mathrm{tGO}$ : thiol-functionalized graphene oxide; TGA: thermogravimetric analysis; gAuNP: glutathione-capped gold nanoparticles; CAuNP: cetyltrimethylammonium bromide-capped gold nanoparticles; cAuNR: cetyltrimethylammonium bromide-capped gold nanorods; EDC: (1-ethyl-3-(3-dimethylaminopropyl)carbodiimide); SP: surface plasmon; GS: glutathione; TEM: transmission electron microscopy; SR: alkanethiolate; THF: tetrahydrofuran.

\section{Authors' contributions}

HP and SL contributed equally on conducting both experimental work related to the synthesis and characterization of nanoparticles, nanoparticle hybrids, and heat-treated hybrids. NW and BW contributed partially for TEM imaging and analysis of nanoparticles and hybrids. YS supervised the project. The manuscript was initially written through contributions of HP and SL and revised by BW and YS. All authors read and approved the final manuscript.

\section{Competing interests}

The authors declare that they have no competing interests.

\section{Funding}

This study was funded by the National Institute of General Medical Science (\#SC3GM089562) of the National Institutes of Health. 


\section{Publisher's Note}

Springer Nature remains neutral with regard to jurisdictional claims in published maps and institutional affiliations.

Received: 18 September 2018 Accepted: 24 December 2018

Published online: 08 January 2019

\section{References}

1. E.S. Kooij, W. Ahmed, H.J.W. Zandvliet, B. Poelsema, Localized plasmon in noble metal nanospheroids. J. Phys. Chem. C 115, 10321-10332 (2011)

2. C. Tan, X. Huang, H. Zhang, Synthesis and applications of graphene-based noble metal nanostructures. Mater. Today 16, 29-36 (2013)

3. R. Muszynski, B. Seger, P.V. Kamat, Decorating graphene sheets with gold nanoparticles. J. Phys. Chem. C 112, 5263-5266 (2008)

4. P. Zhao, N. Li, D. Astruc, State of the art in nanoparticle synthesis. Coord. Chem. Rev. 257, 638-665 (2013)

5. H.S. Bae, E. Seo, S. Jang, K.H. Park, B.-S. Kim, Hybrid gold nanoparticlereduced graphene oxide nanosheets as active catalysts for highly efficient reduction of nitroarenes. J. Mater. Chem. 21, 15431-15436 (2011)

6. X. Zhang, J. Zhang, H. Wang, Y. Hao, X. Zhang, T. Wang, Y. Wang, R. Zhao, H. Zhang, B. Yang, Thermal-induced surface plasmon band shift of gold nanoparticle monolayer: morphology and refractive index sensitivity. Nanotechnology 21, 1-11 (2010)

7. P.K. Jain, X. Huang, I.H. El-Sayed, M.A. El-Sayed, Review of some interesting surface plasmon resonance-enhanced properties of noble metal nanoparticles and their applications to biosystems. Plasmonics $\mathbf{2}$, 107-118 (2007)

8. C. Hu, J. Rong, J. Cui, Y. Yang, L. Yang, Y. Wang, Y. Liu, Fabrication of a graphene oxide-gold nanorod hybrid material by electrostatic self-assembly for surface-enhanced Raman scattering. Carbon 51, 255-264 (2013)

9. E.T. Castellana, R.C. Gamez, D.H. Russell, Label-free biosensing with lipidfunctionalized gold nanorods. J. Am. Chem. Soc. 133, 4182-4185 (2011)

10. P.T. Yin, T.-H. Kim, J.-W. Choi, K.-B. Lee, Prospects for graphene-nanoparticle-based hybrid sensors. Phys. Chem. Chem. Phys. 15, 12785-12799 (2013)

11. W. Hong, H. Bai, Y. Xu, Z. Yao, Z. Gu, G. Shi, Preparation of gold nanoparticle/graphene composites with controlled weight contents and their application in biosensors. J. Phys. Chem. C 2010, 1822-1826 (2010)

12. L. Polavarapu, M. Manna, Q.-H. Xu, Biocompatible glutathione capped gold clusters as one- and two-photon excitation fluorescence contrast agents for live cells imaging. Nanoscale 3, 429-434 (2011)

13. Z. Zhang, L. Sun, Conjugating folic acid to gold nanoparticles through glutathione for targeting and detecting cancer cells. Bioorganic Med. Chem. 18, 5528-5534 (2010)

14. C. Xu, D. Yang, L. Mei, B. Lu, L. Chen, Q. Li, H. Zhu, T. Wang, Encapsulating gold nanoparticles or nanorods in graphene oxide shells as a novel gene vector. ACS Appl. Mater. Interfaces 5, 2715-2724 (2013)

15. Y.-S. Shon, D. Choi, J. Dare, T. Dinh, Synthesis of nanoparticle-cored dendrimers by convergent dendritic functionalization of monolayerprotected nanoparticles. Langmuir 24, 6924-6931 (2008)

16. Y.-S. Shon, H.Y. Choi, M.S. Guerrero, C. Kwon, Preparation of nanostructured film arrays for transmission localized surface plasmon sensing. Plasmonics 4, 95-105 (2009)

17. Z. Nuño, B. Hessler, J. Ochoa, Y.-S. Shon, C. Bonney, Y. Abate, Nanoscale subsurface- and material-specific identification of single nanoparticles. Opt. Express 19, 20865-20875 (2011)

18. Q. Zhuo, Y. Ma, J. Gao, P. Zhang, Y. Xia, Y. Tian, X. Sun, J. Zhong, X. Sun, Facile synthesis of graphene/metal nanoparticle composites via self-catalysis reduction at room temperature. Inorg. Chem. 52, 3141-3147 (2013)

19. S.E. Lohse, J.A. Dahl, J.E. Hutchison, Direct synthesis of large water-soluble functionalized gold nanoparticles using Bunte salts as ligand precursors Langmuir 26, 7504-7511 (2010)

20. H. Ismaili, D. Geng, A.X. Sun, T.T. Kantzas, M.S. Workentin, Light-activated covalent formation of gold nanoparticle-graphene and gold nanoparticle-glass composites. Langmuir 27, 13261-13268 (2011)

21. J. Hrbek, F.M. Hoffmann, J.B. Park, P. Liu, D. Stacchiola, Y.S. Hoo, S. Ma, A. Nambu, J.A. Rodriguez, M.G. White, Adsorbate-driven morphological changes of a gold surface at low temperature. J. Am. Chem. Soc. 130 17272-17273 (2008)

22. M.W. Sugden, T.H. Richardson, G. Leggett, Sub-10 $\Omega$ resistance gold films prepared by removal of ligands from thiol-stabilized $6 \mathrm{~nm}$ gold nanoparticles. Langmuir 26, 4331-4338 (2010)

23. S. Sun, P. Wu, Easy fabrication of macroporous gold films using graphene sheets as a template. ACS Appl. Mater. Interfaces 5, 3481-3486 (2013)

24. H. Pan, S. Low, N. Weerasuriya, Y.-S. Shon, Graphene oxide-promoted reshaping and coarsening of gold nanorods and nanoparticles. ACS Appl. Mater. Interfaces 7, 3406-3413 (2015)

25. E.-S.A.M. Al-Sherbini, UV-visible light reshaping of gold nanorods. Mater. Chem. Phys. 121, 349-353 (2010)

26. M. Gordel, J. Olesiak-Banska, K. Matczyszyn, C. Nogues, M. Buckle, M. Samoc, Post-synthesis reshaping of gold nanorods using a femtosecond laser. Phys. Chem. Chem. Phys. 16, 71-78 (2014)

27. Y. Horiguchi, K. Honda, Y. Kato, N. Nakashima, Y. Niidome, Photothermal reshaping of gold nanorods depends on the passivating layers of the nanorod surfaces. Langmuir 24, 12026-12031 (2008)

28. K.G. Stamplecoskie, Y.-S. Chen, P.V. Kamat, Excited-state behavior of luminescent glutathione-protected gold clusters. J. Phys. Chem. C 118, 1370-1376 (2014)

29. S. Deol, N. Weerasuriya, Y.-S. Shon, Stability, cytotoxicity, and cell uptake of water-soluble dendron-conjugated gold nanoparticles with 3,12, and $17 \mathrm{~nm}$ core. J. Mater. Chem. B 3, 6071-6080 (2015)

30. Z. Wu, J. Suhan, R. Jin, One-pot synthesis of atomically monodisperse, thiol-functionalized $\mathrm{Au}_{25}$ nanoclusters. J. Mater. Chem. 19, 622-626 (2009)

31. S. Kumar, R. Jin, Water-soluble $\mathrm{Au}_{25}\left(\right.$ (Capt) ${ }_{18}$ nanoclusters: synthesis, thermal stability, and optical properties. Nanoscale 4, 4222-4227 (2012)

32. X. Gong, Y. Bi, Y. Zhao, G. Liu, W.Y. Teoh, Graphene oxide-based electrochemical sensor: a platform for ultrasensitive detection of heavy metal ions. RSC Adv. 4, 24653-24657 (2014)

33. J. Huang, L. Zhang, B. Chen, N. Ji, F. Chen, Y. Zhang, Z. Zhang, Nanocomposites of size-controlled gold nanoparticles and graphene oxide: formation and applications in SERS and catalysis. Nanoscale 2, 2733-2738 (2010)

34. N.E.A. Reeler, K.A. Lerstrup, W. Somerville, J. Speder, S.V. Petersen, B.W. Laursen, M. Arenz, X. Qiu, T. Vosch, K. Nørgaard, Gold nanoparticles assembled with dithiocarbamate-anchored molecular wires. Sci. Rep. $\mathbf{5}$ $15273(2015)$

35. H. Yao, L. Jin, H.J. Sue, Y. Sumi, R. Nishimura, Facile decoration of Au nanoparticles on reduced graphene oxide surfaces via a one-step chemical functionalization approach. J. Mater. Chem. A 1, 10783-10789 (2013)

36. A. Ghosh, T. Pradeep, J. Chakrabarti, Coalescence of atomically precise clusters on graphenic surfaces. J. Phys. Chem. C 118, 13959-13964 (2014)

37. P.P.H.C. Cheng, D. Silvester, G. Wang, G. Kalyuzhny, A. Douglas, R.W. Murray, Dynamic and static quenching of fluorescence by $1-4 \mathrm{~nm}$ diameter gold monolayer-protected clusters. J. Phys. Chem. B 110, 4637-4644 (2006)

38. Z. Xia, P. Li, Y. Wang, T. Song, Q. Zhang, B. Sun, Solution-processed gold nanorods integrated with graphene for near-infrared photodetection via hot carrier injection. ACS Appl. Mater. Interfaces 7, 24136-24141 (2015)

39. Y.-S. Shon, M. Aquino, T.V. Pham, D. Rave, M. Ramirez, K. Lin, P. Vaccarello, G. Lopez, T. Gredig, C. Kwon, Stability and morphology of gold nanoisland arrays generated from layer-by-layer assembled nanoparticle multilayer films: effects of heating temperature and particle size. J. Phys. Chem. C 115, 10597-10605 (2011)

40. S.R. Isaacs, H. Choo, W.-B. Ko, Y.-S. Shon, Chemical, thermal, and ultrasonic stability of hybrid nanoparticles and nanoparticle multilayer films. Chem. Mater. 18, 107-114 (2006)

41. P. Vaccarello, L. Tran, J. Meinen, C. Kwon, Y. Abate, Y.-S. Shon, Characterization of localized surface plasmon resonance transducers produced from $\mathrm{Au}_{25}$ nanoparticle multilayers. Colloids Surf. A 402, 146-151 (2012)

42. M. Kim, C. Lee, J. Jang, Fabrication of highly flexible, scalable, and highperformance supercapacitors using polyaniline/reduced graphene oxide film with enhanced electrical conductivity and crystallinity. Adv. Funct. Mater. 24, 2489-2499 (2014) 